\title{
Suppression of Bragg Scattering by Collective Interference of Spatially Ordered Atoms with a High- $Q$ Cavity Mode
}

\author{
Stefano Zippilli, ${ }^{1}$ Giovanna Morigi, ${ }^{1}$ and Helmut Ritsch ${ }^{2}$ \\ ${ }^{1}$ Abteilung für Quantenphysik, Universität Ulm, Albert-Einstein-Allee 11, D-89069 Ulm, Germany \\ ${ }^{2}$ Institut für Theoretische Physik, Universität Innsbruck, Technikerstrasse 25/2, A-6020 Innsbruck, Austria
}

(Received 3 February 2004; published 17 September 2004)

\begin{abstract}
When $N$ driven atoms emit in phase into a high- $Q$ cavity mode, the intracavity field generated by collective scattering interferes destructively with the pump driving the atoms. Hence atomic fluorescence is suppressed and cavity loss becomes the dominant decay channel for the whole ensemble. Microscopically, 3D light-intensity minima are formed in the vicinity of the atoms that prevent atomic excitation and form a regular lattice. The effect gets more pronounced for large atom numbers, when the sum of the atomic decay rates exceeds the rate of cavity losses and one would expect the opposite behavior. These results provide new insight into recent experiments on collective atomic dynamics in cavities.
\end{abstract}

Recent experimental progress has allowed one to trap cold atomic gases within high- $Q$ optical resonators. In ring resonators collective oscillations of the atoms have been reported [1] and strong accelerations were demonstrated [2]. In pioneering experiments with transversally pumped atoms in a standing-wave cavity very efficient cooling of large ensembles was achieved by Vuletic and co-workers $[3,4]$. They found strong coherent emission into the cavity mode, which exceeded the rate of scattering into free space by orders of magnitude $[3,4]$. The cavity field randomly attained one of two well defined phases with $\pi$ difference. This behavior gives strong evidence of formation of a regular atomic pattern with wavelength periodicity, so that the atoms scatter in phase into the cavity mode, while the observed $\pi$-phase jumps correspond to transitions to an alternative pattern shifted by half wavelength. This hypothesis is supported by numerical results [5], showing self-organization into the pattern in the parameter regime of $[3,4]$. In this context, the enhanced rate of cavity emission has been interpreted as a signature of Bragg scattering. However, Bragg scattering by itself does not explain the dramatic suppression of atomic fluorescence, which makes cavity decay the dominant channel of dissipation.

In this Letter, we argue that the origin of the enhanced cavity emission rate and the suppression of atomic fluorescence can be traced back to quantum interference of coupled oscillators, namely, the field mode and the collective atomic polarization. This model reproduces qualitatively several features of the stationary dynamics observed in [4], even though in the experiment the atoms had a more complex internal structure and more than a single mode was involved. It predicts that, when the atoms are organized in a regular spatial pattern emitting in phase into the cavity mode, stationary cavity field and pump have opposite phases and mutually cancel at the atomic sites. Thus, the atoms are not excited and fluores- cence, as well as superradiant scattering into the cavity mode, are suppressed. These dynamics are found in high- $Q$ resonators and differ radically from the dynamics at the basis of cavity-enhanced emission observed in experiments with a similar setup but in the bad-cavity limit regime [6]. As in optical bistability [7], the effect occurs in the strong coupling regime, but it is characterized by only one steady state, for which the atoms are in the ground state. In this case, the amplitude of the cavity field canceling the pump field is independent of the number of atoms. Hence with properly rescaled parameters the effect can also be found for a single atom. For many atoms efficient suppression of excitation is present only when they emit in phase into the cavity mode. The suppression of fluorescence is accompanied by a coherent cavity field, which gives a clear signature of pattern formation. This interpretation is supported by an analysis showing that the regular spacing of the atoms is a stable configuration in the parameter regime of [4].

We consider a single standing-wave cavity mode resonantly coupled to $N$ atomic dipoles with spatially dependent coupling constant $g=g_{0} \cos (2 \pi x / \lambda)$, where $\lambda$ is the mode wavelength and $x$ gives the position along the cavity axis. In addition, the dipoles couple with Rabi frequency $\Omega$ to a plane-wave field at frequency $\omega_{L}$ propagating orthogonal to the cavity axis, as illustrated in Fig. 1. Here we assume that the atoms are pointlike and distributed at the positions $x_{n}^{(0)}=x+n \lambda$, where $n$ is an integer such that $g\left(x_{n}^{(0)}\right)=g \neq 0$. This assumption will be later justified by showing that this is in fact a mechanically stable situation. The pattern is assumed to have a low filling factor, such that collective radiative effects in free space are negligible. The coherent dynamics of the system is described by the Hamiltonian $H=-\hbar \delta_{c} a^{\dagger} a+$ $\sum_{n=1}^{N} H_{n}$, with $a, a^{\dagger}$ annihilation and creation operators of a cavity photon, and 


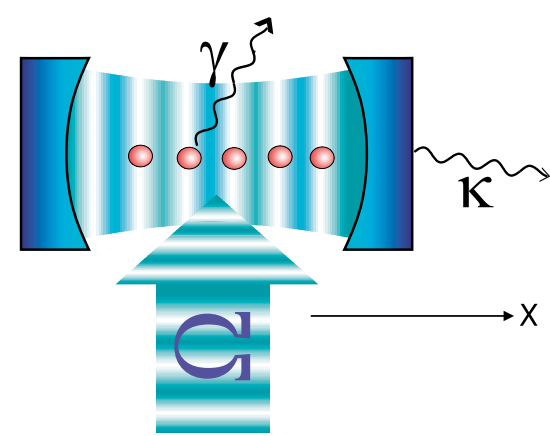

FIG. 1 (color online). $\quad N$ atoms are inside a $1 \mathrm{D}$ optical resonator and homogeneously driven by a laser propagating in the transverse direction. The atoms are localized according to a spatial pattern such that they emit in phase into the cavity mode (see [5]).

$$
H_{n}=\hbar \Delta \sigma_{n} \sigma_{n}^{\dagger}+\hbar\left[\sigma_{n}^{\dagger}\left(g\left(x_{n}\right) a+\Omega\right)+\text { H.c. }\right],
$$

with $\sigma_{n}, \sigma_{n}^{\dagger}$ dipole operators for the $n$th atom, and $\delta_{c}=$ $\omega_{L}-\omega_{c}, \Delta=\omega_{L}-\omega_{0}$, detunings of the laser from the frequency of the cavity and of the dipole, respectively. The master equation for the density matrix $\rho$ of atoms and cavity mode is $\frac{\partial}{\partial t} \rho=[H, \rho] / i \hbar+\mathcal{L} \rho+\mathcal{K} \rho$, where the superoperator $\mathcal{L}$ describes damping due to spontaneous decay at rate $\gamma$, while $\mathcal{K} \rho$ describes cavity decay with zero-photon linewidth $\kappa$ [8]. Note that for the moment we consider a one-dimensional model and neglect the center-of-mass motion.

In the parameter regime when the collective atomic dipole is driven below saturation, for $|\gamma / 2+i \Delta| \gg$ $\sqrt{N} g, \sqrt{N} \Omega$, the stationary field amplitude takes the form

$$
\alpha=-\frac{\Omega}{g_{0}} \frac{N s(\gamma / 2+i \Delta)}{N s(\gamma / 2+i \Delta)+\kappa / 2-i \delta_{c}},
$$

and the occupation of the excited state of each atom is

$$
\Pi_{n}=\frac{\Omega^{2}}{(\gamma / 2)^{2}+\Delta^{2}} \frac{\kappa^{2} / 4+\delta_{c}^{2}}{(N s \gamma+\kappa)^{2} / 4+\left(N s \Delta-\delta_{c}\right)^{2}},
$$

where $s=g^{2} /\left(\Delta^{2}+\gamma^{2} / 4\right)$. In Eqs. (2) and (3) the number of atoms $N$ scales the terms containing the atomic parameters. In particular, for $N$ sufficiently large the occupation of the atomic excited state vanishes as $1 / N^{2}$, while the field inside the cavity tends to the constant value $\alpha_{0}=-\Omega / g$, which neither depends on the detunings $\Delta, \delta_{c}$, nor on the cavity and atomic decay rates. This behavior is visible in Fig. 2, where the total fluorescence intensity $I_{\text {at }}=N \gamma \Pi_{n}$ and the signal at the cavity mirror $I_{\text {cav }}=\kappa|\alpha|^{2}$ are plotted as a function of $N$. Here a threshold value $N_{0}$ can be identified that separates two different dynamics, corresponding to the regimes of weak and strong coupling. In fact, for $N \ll N_{0}$ the excited state population in (3) is approximately given by the value in free space, and the field intensity scales quadratically with the number of atoms: There is no backaction of the
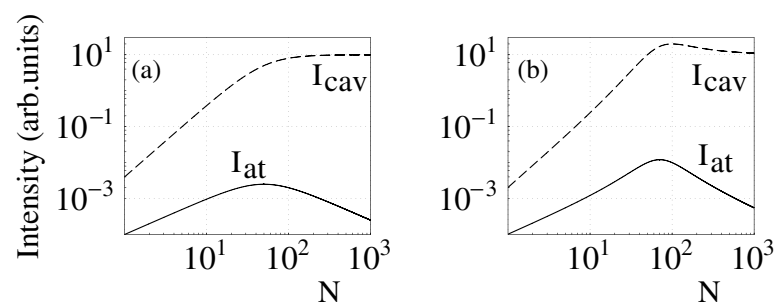

FIG. 2. Intensity of the signal at the cavity mirror ( $I_{\text {cav }}$, dashed lines) and total atomic fluorescence intensity $\left(I_{\text {at }}\right.$, solid lines) as a function of $N$ for $\Omega=g=10 \gamma, \kappa=10 \gamma, \Delta=$ $-1000 \gamma$, and (a) $\delta_{c}=0$, (b) $\delta_{c}=-5 \gamma$.

cavity on the atomic dynamics, since the cavity decay rate is faster than the rate at which the atomic degrees of freedom reach their steady state. This is the regime where Bragg enhancement of superradiant scattering into the cavity mode is found. For $N \gg N_{0}$, on the other hand, the total power dissipated by spontaneous emission scales with $1 / N$. Thus the system dissipates mainly through cavity loss, where the signal at the cavity output is constant and, remarkably, independent of $N$. Hence for $N \gg$ $N_{0}$ there is no signature of Bragg scattering. At $\Delta=0$ this regime corresponds to a large cooperativity parameter $C=N g_{0}^{2} / \gamma \kappa>1$. A situation closely related to the experimental parameters of [4] is found for the case $|\Delta| \gg$ $\gamma, \kappa$ and $\delta_{c} \neq 0$, illustrated in Fig. 2(b). Here the critical number of atoms $N_{0} \sim\left|\delta_{c} \Delta\right| / g_{0}^{2}$ denotes the situation when the laser drives resonantly the collective resonance of the atoms and cavity system, manifesting itself in the enhancement of the two signals visible in Fig. 2(b). When the limit $N \gg N_{0}$ is reached, on the other hand, the system behaves as in the resonant case; namely, the cavity field is independent of the number of atoms and the atoms do not fluoresce.

These results can be understood in the limiting case $\kappa=0$ and $\delta_{c}=0$. For these values the dependence on $N$ drops out from Eqs. (2) and (3) and one obtains $\alpha=\alpha_{0}$ and $\Pi_{n}=0$. In this case, the dynamics are scalable down to a single atom and are exactly solvable [9]. Here the steady state $\rho_{\text {st }}$ is a pure state, with the cavity field in the coherent state at the amplitude $\alpha_{0}$ and the atom in the ground state. Thus, no fluorescence photons are emitted. This behavior is due to destructive interference between the pump and cavity field that drive the atom with equal intensity but opposite phase, as one can verify by applying the Hamiltonian (1) to the system prepared in $\rho_{\text {st }}$. These dynamics are independent of the intensity of the pump, which determines only the mean number of cavity photons at steady state through the ratio $\Omega / g$ [10].

Several features of these dynamics largely survive for finite and fairly large values of the cavity damping $\kappa$. In particular, the signal transmitted at the cavity mirror $I_{\text {cav }}=\kappa \operatorname{Tr}\left\{a^{\dagger} a \rho\right\}$ can be orders of magnitude larger than the fluorescence signal $I_{\text {at }}=\gamma \operatorname{Tr}\left\{\sigma^{\dagger} \sigma \rho\right\}$, as illus- 

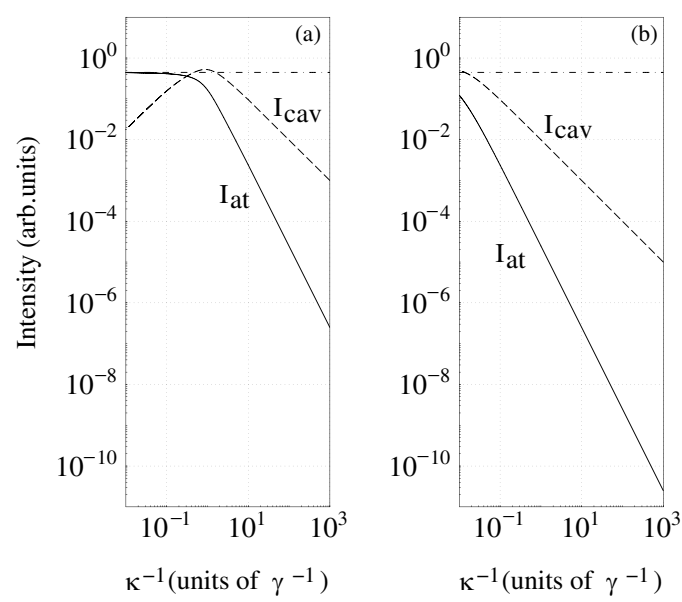

FIG. 3. Intensity of the signal at the cavity mirror $\left(I_{\text {cav }}\right.$, dashed line) and of the atomic fluorescence signal $\left(I_{\text {at }}\right.$, solid line) as a function of $\kappa^{-1}$ for a single atom. Here $\Omega=\gamma, \Delta=$ $\delta_{c}=0$, and (a) $g=\gamma$, (b) $g=10 \gamma$. The dash-dotted line corresponds to the free space fluorescence rate.

trated in Fig. 3. In the limits $\Delta=0$ and $g \gg \gamma \gg \kappa$, these signals have the form

$$
I_{\mathrm{at}} \approx \kappa\left|\alpha_{0}\right|^{2} / 8 C_{1}, \quad I_{\mathrm{cav}} \approx \kappa\left|\alpha_{0}\right|^{2}\left(1-1 / 8 C_{1}\right),
$$

where $C_{1}=g^{2} / 2 \gamma \kappa$ is the cooperativity parameter per atom [11]. Hence, the regime of small $\kappa$ in Fig. 3 corresponds to a large cooperativity parameter, such as the regime of large $N$ in Fig. 2. For $C_{1} \gg 1$ the field inside the cavity is still well approximated by a coherent state, as it can be verified from the second-order correlation function $g^{(2)}(0)$ at the cavity mirror. In Fig. 4(b), $g^{(2)}(0)$ is plotted as a function of $\kappa^{-1}$, showing that the cavity field exhibits a Poissonian behavior for a fairly large range of values of $\kappa$ and even for a very small number of photons inside the cavity (solid lines in Fig. 4). Thus the cavity mode is in a coherent state, independent of the mean energy of the cavity field. This behavior contrasts dramatically with the antibunching observed when the pump is set directly on the cavity [11,12].

Further insight is gained from the rate of photon scattering obtained by scanning an additional weak transverse pump across atomic resonance. Denoting with $\delta_{P}=\omega_{P}-\omega_{c}$, the detuning of the probe from the cavity frequency, for $\delta_{c}=0$ and $\kappa=0$ the atom scatters photons at the rate

$$
w\left(\delta_{P}\right) \propto \frac{\gamma \delta_{P}^{2}}{\left[\delta_{P}\left(\delta_{P}+\Delta\right)-g(x)^{2}\right]^{2}+\delta_{P}^{2} \gamma^{2} / 4},
$$

which vanishes at $\delta_{P}=0$, thereby exhibiting a Fano-like profile at this point $[13,14]$. It is remarkable that the pump intensity $\Omega$, and thus the mean number of cavity photon, does not appear in Eq. (4). In particular, the positions of the two maxima of $w\left(\delta_{P}\right)$ correspond to the energies of the dressed states of the atom in an empty cavity. This
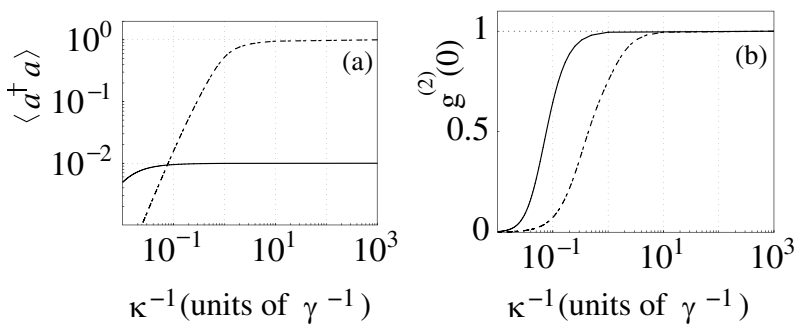

FIG. 4. (a) Mean number of cavity photon and (b) secondorder correlation function $g^{(2)}(0)$ as a function of $\kappa^{-1}$ for $\Delta=$ $\delta_{c}=0, \Omega=\gamma$, and $g=\gamma$ (dashed line), $g=10 \gamma$ (solid line).

behavior, which is independent of the cavity-field energy, is due to the vanishing electric field at the atomic position.

In the limits $\kappa=0$ and $\delta_{c}=0$, these results can be scaled with the number $N$ of atoms. Then, the Hamiltonian $H$ describes the Jaynes-Cunning dynamics of a collective dipole coupling to the cavity mode with $g \rightarrow \sqrt{N} g$, provided that the atoms are localized and distributed according to the spatial pattern. At steady state, when the collective dipole is driven below saturation, the atoms are in the ground state and the cavity field is a coherent state of amplitude $\alpha_{0}$ [15], while the splitting of the maxima in $w\left(\delta_{P}\right)$ scales according to the rule $g \rightarrow \sqrt{N} g$. Note that the scaling can be applied only in this ideal case, where one has always strong coupling. For bad cavities and $\delta_{c} \neq 0$, on the other hand, only a large number of atoms allows one to achieve the necessary large cooperativity for accessing these dynamics. In contrast to optical bistability, however, here no bistable behavior is found as the atoms are in the ground state.

In Fig. 5 the ratio $I_{\text {cav }} / I_{\text {at }}$ is displayed for two atoms at the positions $x_{1}$ and $x_{2}$, showing clearly that this ratio is maximum when the atoms are a wavelength apart. For $\kappa \neq 0$, absolute maxima are found when the atoms are at the antinodes of the cavity mode, where the cooperativity parameter is largest and the total electric field practically vanish. The three dimensional pattern is found taking into account the phase of the pump. Then, the zeros of the electric field are distributed according to a bodycentered-cubic lattice with distance $\lambda / 2$ between adiacent planes [4,5]. Fluorescence is suppressed when the atoms are localized at these points, thus forming a stationary pattern. This latter condition constitutes a substantial difference to the collective scattering via acceleration observed in the dynamics of the collective atomic recoil laser $[2,16]$.

We study now the mechanical stability of the atomic pattern, which is a central ingredient in our model. We use the coupled semiclassical equations for field and atomic motion [5] to describe the parameter regime of [4], where the atoms are driven at far-off resonance and the relative fluctuations of the cavity-field amplitude are small, and assume $N \gg N_{0}$, so that at leading order in $N$ the cavityfield amplitude is $\alpha_{0}$. A trivial equilibrium configuration 

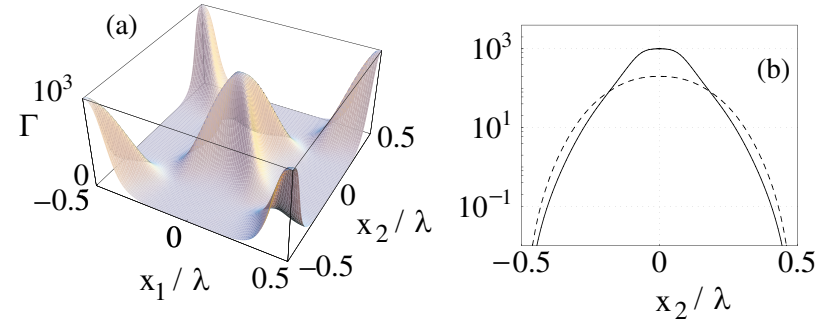

FIG. 5 (color online). (a) Ratio $\Gamma=I_{\text {cav }} / I_{\text {at }}$ for two atoms as a function of their position $x_{1}$ and $x_{2}$ inside the cavity. Here, $g_{0}=10 \gamma, \Delta=100 \gamma, \delta_{c}=0, \Omega=\gamma$ for both atoms, and $\kappa=$ $0.2 \gamma$. (b) Ratio as in (a) as a function of $x_{2}$ for $x_{1}=0$. The dashed line shows $\Gamma$ for the same parameters, but $\kappa=\gamma$. Note that $x_{1}, x_{2}$ are plotted modulus $\lambda$, and $x_{1} \neq x_{2}$.

is found when the atoms are at the antinodes of the cavity mode with spacing equal to the wavelength $\lambda$. Stability for small fluctuations $\delta x_{n}$ of the atomic positions is found when the first derivative of the semiclassical force at these points is negative, which corresponds to the condition [17]

$$
\delta f_{n} \sim 2 \hbar k^{2}\left(\Omega / g_{0}\right)^{2} \delta_{c} / N<0,
$$

that is, $\delta_{c}<0$. Notably the stability condition is not affected by the sign of the detuning $\Delta$. This behavior has been verified numerically. At these points the intensity of the force $\delta f_{n} \delta x_{n}$ is proportional to the average number of cavity photons and thus to the pump intensity. Hence, the mechanical potential at these positions gets steeper for larger pumps. This dependence is consistent with the threshold behavior measured in $[3,4]$. Also the dependence on the detunings is in line with the experimental observations, which found enhanced cavity emission for both signs of the detuning $\Delta$ but for a finite range of values of $\delta_{c}<0[3,4]$. It is an open and intriguing question how the system evolves into the self-organized pattern and how the nonequilibrium dynamics depend on the various parameters. Moreover, do other (meta-) stable equilibrium states exist? And how does noise affect the pattern stability? Such questions can be addressed using the theory developed in [18].

For systems of one or few atoms in high- $Q$ cavities this interference effect has numerous potential applications. As the atoms generate a position dependent field without being excited, this could be a new version of nondestructive single atom detection. In particular, molecules with no closed cycle could be efficiently detected. Further possibilities are implementations of conditional coherent dynamics as needed for quantum information processing. Here one expects less decoherence as the atoms interact while being almost in the ground state. Scattering from internal atomic superposition states would immediately create entanglement, even in the steady state. Moreover, the coherence properties of the transmitted signal, which are preserved even for very small photon numbers, suggest an alternative kind of photon emitters to the one investigated in $[19,20]$. These investigations may be realized with present experimental setups, which can trap single or few atoms and couple them to the cavity field in a controlled way [21-25].

The authors gratefully acknowledge discussions with H. J. Carmichael, A. Kuhn, H. Mabuchi, G. Rempe, and W. Schleich. This work has been supported by the EUnetworks QUEST and QGATES.

[1] B. Nagorny et al., Phys. Rev. Lett. 91, 153003 (2003).

[2] D. Kruse et al., Phys. Rev. Lett. 91, 183601 (2003).

[3] H.W. Chan et al., Phys. Rev. Lett. 90, 063003 (2003).

[4] A. T. Black et al., Phys. Rev. Lett. 91, 203001 (2003).

[5] P. Domokos and H. Ritsch, Phys. Rev. Lett. 89, 253003 (2002).

[6] D. J. Heinzen et al., Phys. Rev. Lett. 58, 1320 (1987); D. J. Heinzen and M. S. Feld, ibid. 59, 2623 (1987).

[7] R. Bonifacio and L. A. Lugiato, Opt. Commun. 19, 172 (1976); Phys. Rev. A 18, 1129 (1978); L. A. Lugiato, in Progress in Optics, edited by E. Wolf (North-Holland, Amsterdam, 1984), Vol. XXI, p. 69.

[8] H. J. Carmichael, An Open Systems Approach to Quantum Optics (Springer-Verlag, Berlin, 1993).

[9] P. M. Alsing et al., Phys. Rev. A 45, 1793 (1992).

[10] Alternatively, for fixed pump intensity the mean energy of the field depends on the atomic position through $g(x)$.

[11] H. J. Kimble, in Cavity Quantum Electrodynamics, edited by P. R. Berman (Academic Press, New York, 1994), p. 203.

[12] R. Brecha et al., Phys. Rev. A 59, 2392 (1999).

[13] B. Lounis and C. Cohen-Tannoudij, J. Phys. II (France) 2, 579 (1992).

[14] P. R. Rice and R. J. Brecha, Opt. Commun. 126, 230 (1996).

[15] At saturation and for large filling factors dipole-dipole interaction may modify substantially the dynamics. See, for example, J. P. Clemens et al., Phys. Rev. A 68, 023809 (2003).

[16] R. Bonifacio and L. DeSalvo, Nucl. Instrum. Methods Phys. Res., Sect. A 341, 360 (1994); R. Bonifacio et al., Phys. Rev. A 50, 1716 (1994).

[17] This condition is sufficient, since the field amplitude $\alpha$ does not vary in first order in $\delta x_{n}$, nor does the force for small fluctuations in $\alpha$.

[18] P. Domokos and H. Ritch, J. Opt. Soc. Am. B 20, 1098 (2003).

[19] A. Kuhn et al., Phys. Rev. Lett. 89, 067901 (2002).

[20] J. McKeever et al., Nature (London) 425, 268 (2003).

[21] J. A. Sauer et al., Phys. Rev. A 69, 051804 (2004).

[22] J. McKeever et al., Phys. Rev. Lett. 90, 133602 (2003).

[23] J. Eschner et al., Nature (London) 413, 495 (2001).

[24] G. R. Guthöhrlein et al., Nature (London) 414, 49 (2001).

[25] A. B. Mundt et al., Phys. Rev. Lett. 89, 103001 (2002). 